\title{
Philosophiques
}

\section{Du jeu et du sommeil à la gravité et à l'insomnie}

\section{Simonne Plourde}

Volume 13, numéro 2, automne 1986

URI : https://id.erudit.org/iderudit/203322ar

DOI : https://doi.org/10.7202/203322ar

Aller au sommaire du numéro

Éditeur(s)

Société de philosophie du Québec

ISSN

0316-2923 (imprimé)

1492-1391 (numérique)

Découvrir la revue

Citer cet article

Plourde, S. (1986). Du jeu et du sommeil à la gravité et à l'insomnie. Philosophiques, 13(2), 305-332. https://doi.org/10.7202/203322ar

\section{Résumé de l'article}

Emmanuel Lévinas rompt avec les ontologies traditionnelles et porte sa réflexion « au-delà de l'Essence ». Cette rupture avec la totalité de l'Être est nécessitée par l'épiphanie du Visage d'Autrui. L'irréductibilité de l'altérité place d'emblée le sujet au coeur de la relation éthique. Le langage doit s'inventer, s'amplifier, s'inquiéter pour signifier l'Autrement qu'Être ; l'emploi de la métaphore s'avère essentiel pour cette pensée a-typique. On suivra ici le fil de quatre métaphores formant deux couples antithétiques : jeu et gravité, sommeil et insomnie. L'analyse conduira au coeur de la philosophie d'Emmanuel Lévinas, à l'insomnie de la responsabilité pour Autrui.
Ce document est protégé par la loi sur le droit d'auteur. L'utilisation des services d'Érudit (y compris la reproduction) est assujettie à sa politique d'utilisation que vous pouvez consulter en ligne.

https://apropos.erudit.org/fr/usagers/politique-dutilisation/ 


\title{
DU JEU ET DU SOMMEIL A LA GRAVITÉ ET À L'INSOMNIE
}

\author{
par Simonne Plourde
}

\begin{abstract}
RÉSUMÉ. Emmanuel Lévinas rompt avec les ontologies traditionnelles et porte sa réflexion "au-delà de l'Essence». Cette rupture avec la totalité de l'Être est nécessitée par l'épiphanie du Visage d'Autrui. L'ir réductibilité de l'altérité place d'emblée le sujet au cœur de la relation éthique. Le langage doit s'inventer, s'amplifier, s'inquiéter pour signifier l'Autrement qu'Être ; l'emploi de la métaphore s'avère essentiel pour cette pensée a-typique. On suivra ici le fil de quatre métaphores formant deux couples antithétiques: jeu et gravité, sommeil et insomnie. L'analyse conduira au cour de la philosophie d'Emmanuel Lévinas, à l'insomnie de la responsabilité pour Autrui.
\end{abstract}

ABSTRACT. Emmanuel Lévinas breaks with traditional ontologies and carries his reflection «beyond the Essence». This rupture with the wholeness of Being is required by the epiphany of the Other's face. The irreducibility of the otherness places directly the subject in the midst of the ethical relationship. Language has to be invented, to be amplified in order to mean "l'Autrement qu'Être"; the use of the metaphor appears to be essential for this a-typical thinking. Four metaphors composed by two anthetical sets : play and seriousness, sleep and insomnia, are presented here. The analysis will lead to the center of Emmanuel Levinas' philosophy, to the insomnia of the responsibility for the Other.

Je veux dire qu'une vie vraiment humaine ne peut rester vie satis-faite dans son égalité à l'être, vie de quiétude, qu'elle s'éveille à l'autre, c'est-à-dire est toujours à se. dégriser, que l'être n'est jamais - contrairement à ce que disent tant de traditions rassurantes - sa propre raison d'être, que le fameux conatus essendi n'est pas la source de tout droit et de tout sens.

Emmanuel Lévinas *

"E. LÉvinas, Ethique et Infini. Paris, Fayard et Radio-France, 1982, p. 131-132. 
Emmanuel Lévinas n'appartient pas à la génération de ceux qui s'appliquent à conserver la puérilité des idées apprises ou reçues. Dans le rayonnement direct des grands de la pensée, tels un Husserl ou un Heidegger, il a pu à la fois s'exposer à l'éblouissement de leur génialité et prendre ses distances pour déceler dans leur philosophie les limites et les failles d'un Savoir par ailleurs des plus remarquables. Côtoyer les maîtres, en instaurer une critique lucide et prendre discrètement place parmi eux : voilà le destin qui a été réservé à Emmanuel Lévinas.

La pensée lévinassienne a des attaches que le philosophe n'a jamais celées: Platon, parce qu'il a mis le Bien au-dessus des essences; Descartes, parce qu'il a pensé l'Infini ; Kant, parce qu'il a secoué le sommeil dogmatique de la philosophie; Husserl, parce qu'il a fourni la méthode; Heidegger, à la fois admiré et impardonné, parce qu'il a décrit d'une façon incomparable l'angoisse humaine, et initié l'entendement aux sonorités particulières de l'être entendu comme verbe plutôt que comme substantif.

La dette est grande et maintes fois reconnue envers Husserl et Heidegger. Mais, très tôt, Lévinas respire mal à son aise au sein d'une tradition philosophique qui situe le lieu naturel du sensé et l'origine de l'interrogation philosophique dans le psychisme humain entendu comme savoir. Un savoir qui consacre la priorité du théorétique en tentant « une synthèse universelle, une réduction de toute l'expérience, de tout ce qui est sensé, à une totalité où la conscience embrasse le monde, ne laisse rien d'autre hors d'elle, et devient ainsi pensée absolue. La conscience de soi est en même temps la conscience du tout ${ }^{1}$.

Lévinas acquiesce à la première contestation radicale de la priorité du théorétique faite par Husserl et se sent un peu plus à l'aise avec l'intentionnalité axiologique, même si cette dernière se confine en définitive dans la représentation. Mais c'est Franz Rosenzweig qui met Lévinas sur la piste d'une critique radicale de la totalité. À la suite de cet autre maître vénéré, Lévinas s'attachera à faire éclater la totalité du savoir et de la conscience, à penser autrement le rationnel. «Brisure de l'universalité de la

1. Ibid. p. 79. 
raison théorétique qui s'était tôt levée dans le "Connais-toi toimême" pour rechercher l'univers entier dans la conscience de soi» ${ }^{2}$. Plus importante et plus véridique que la métaphysique traditionnelle du sujet où l'être se présente en totalité, sera le débordement de la subjectivité par l'idée de l'Infini ; car Autrui comme Visage est là, quotidien, irréductible, irrécusable, dans la proximité d'un face à face et non d'une latéralité, «comme une mise en question incessante, sans ultimité, de la priorité et de la quiétude du Même, telle la brûlure sans consumation d'une flamme inextinguible» ${ }^{3}$.

Dans l'obligation à l'égard d'Autrui, dans une responsabilité incessible antérieure à tout choix librement consenti, Emmanuel Lévinas découvre le SENS, la SIGNIFICATION première. Alors que le savoir est recherche d'une adéquation avec l'être même qu'il absorbe dans l'immanence d'un sujet, la relation au visage est d'emblée éthique et transcendance. Cette transcendance éthique dicte la thèse majeure de Lévinas: «la socialité est un rapport tout autre que celui qui s'établit dans la connaissance et (...) la socialité, elle-même, est commandée par la Parole de Dieu qui est le visage d'autrui» ${ }^{4}$.

Cette sortie de l'être conçu en tant que corrélatif d'un savoir, cet «autrement qu'être» pose à Lévinas une question ultime - dont l'écho se répercute à travers l'œuvre entière - qui rend problématique ce qui est pour tous le plus naturel: «Est-ce que j'ai droit d'être? Est-ce qu'étant dans le monde, je ne prends pas la place de quelqu'un? Mise en question de la persévérance, naïve et naturelle, dans l'être! ${ }^{5}$.

Une mise en question de l'être aussi radicale marque l'œuvre d'Emmanuel Lévinas d'une originalité qui déconcerte. Originalité du questionnement; originalité des thèses, en particulier de celle qui soutient que l'éthique est philosophie première; originalité du style lui-même qui a dû s'inventer, s'amplifier, se surpasser,

2. E. LÉvinas. "Paix et Proximité» dans Les Cabiers de la Nuit surveillée. No 3, Lagrasse, Ed. Verdier, 1984, p. 340.

3. E. Levinas. De Dieu qui vient à l'Idée. Paris, Vrin, 1982, p. 60-61.

4. E. LEvinas. Transcendance et Intelligibilité. Genève, Labor et Fides, 1984, p. 53.

5. E. LEvinAs. Éthique et Infini. Op. cit., p. 131. Voir l'une des citations en exergue dans Autrement qu'être... p. VI : « ... C'est là ma place au soleil. Voilà le commencement et l'image de l'usurpation de toute la terre». (Pascal, Pensées). 
voire se torturer, se triturer, s'in-quiéter pour faire dé-couvrir le plus dans le moins, pour signifier l'autrement qu'être. Recours à l'emphase, à la métaphore, à la désarticulation des vocables, aux mots reliés par des traits d'union pour signifier des ensembles quasi indicibles : voilà ce qui caractérise la parole qui épouse dans tous ses méandres une pensée déjà a-typique ${ }^{6}$. À ce style qui, en tentant d'instaurer et de percer l'«autrement qu'être», frise souvent le poétique, convient l'éloge que Lévinas lui-même décerne à l'écriture du poète Paul Celan:

Ne suggère-t-il pas la poésie elle-même comme une modalité inouie de l'autrement qu'être? (...) L'inéludable: l'interruption de l'ordre ludique du beau et du jeu des concepts et du jeu $d u$ monde; l'interrogation de l'Autre, recherche de l'Autre. Recherche se dédiant en poème à l'autre : un chant monte dans le donner, dans l'un-pourl'autre, dans la signifiance même de la signification. Signification plus ancienne que l'ontologie et la pensée de l'être et que supposent savoir et désir, philosophie et libido 7 .

Mon propos n'aura pas l'audace de s'attaquer à l'ensemble de la stylistique lévinassienne. Là n'est point mon intérêt primordial. Je me limiterai à l'examen de quatre métaphores qui forment deux couples antithétiques, à savoir JEU et GRAVITÉ, SOMMEIL et INSOMNIE. Il ne s'agit nullement de "dégonfler l'enflement des métaphores». Car, l'emploi de la métaphore n'est pas chez Lévinas un procédé esthétique; il est en effet requis par la gravité du réel auquel le Moi (ou le Même) est exposé. Par le biais du langage, l'analyse devrait nous introduire à une "absence», au cœur de la question de l'«autrement qu'être»; elle aura atteint son but si elle réussit à secouer la quiétude ensommeillée des êtres que peut-être nous sommes, "ayant bonne assise ou reposant sur la solidité sous-jacente de [notre] substance ${ }^{8}$, pour nous réveiller de la présence et de l'être et nous convoquer à une interminable insomnie.

6. Voir une excellente étude du style de Lévinas dans Exercices de la patience. $\mathbf{N}^{\circ} 1$, Paris, «Obsidiane», 1980 : Francis Wybrands. «Vers une signifiance hors contexte», p. 51-56.

7. E. LEvinas. Noms propres. Montpellier, Fata Morgana, 1976, p. $65-66$

8. E. LÉvinas. "Paix et Proximité», dans Les Cabiers de la Nuit surveillée. Op. cit., p. 339. 
Abordons dans un premier temps les métaphores du jeu et du sommeil. Un second point examinera celles de la gravité et de l'insomnie.

\section{LES DISTRACTIONS DU MÊME: LE JEU ET LE SOMMEIL}

Husserl, a-t-il été dit plus haut, a fourni la méthode. Fidèle à la phénoménologie, Emmanuel Lévinas a éprouvé la finesse de cet instrument d'analyse et l'a manié avec dextérité dès le premier exposé de sa pensée personnelle dans un petit livre paru en 1947 : De l'existence à l'existant. À l'occasion de l'analyse de l' "il y a», - le phénomène de l'être impersonnel, le «il», être anonyme qu'aucun étant ne revendique - Lévinas éclaire d'autres modalités de l'être (ce dernier pris dans son sens verbal), à savoir: la fatigue, la paresse, l'effort, le sommeil, l'insomnie, etc.

C'est dans cet ouvrage qu'on ira chercher en premier lieu le concept de jeu, qu'on mettra en lumière ses implications, afin de percevoir ce qui perce sous cette métaphore qu'on voit courir à travers l'œuvre lévinassienne: JEU dans nos relations avec le monde; JEU de la pensée qui re-présente; JEU ambigu de la sensibilité.

\section{La métaphore du JEU}

\section{A. Le concept de jeu et ses implications}

Le jeu s'insère comme attitude dans la trame de notre quotidien. Laissons d'abord la parole à Lévinas:

Le jeu commence aussi, mais son commencement manque de sérieux. Il est la légèreté même. On peut, à tout instant, en tirer son épingle. Il se compose de gestes, de mouvements, de décisions, de sentiments, autant d'actes qui commencent, mais sa réalité de jeu se situe au-dessus de cette base et est essentiellement faite d'irréalité. C'est pourquoi la réalité scénique - et chose remarquable, cela ne s'est jamais dit ni d'un poème, ni d'un tableau - a toujours été interprétée comme jeu. En tant que réalité, elle ne laisse pas de traces. Le néant qui la précède est égal à celui qui la suit. Ses événements n'ont pas de vrai temps. Le jeu n'a pas d'histoire. Il est cette existence paradoxale qui ne se prolonge pas en avoir. L'instant du jeu est, mais il ne tient pas à lui-même. Il n'entretient pas avec lui-même une relation de possession. 
Il n'a rien, ne lègue rien après son évanouissement, sombrant «armes et bagages » dans le néant. Et il peut si magnifiquement finir parce qu'il n'a jamais commencé pour de bon?

Ainsi décapée par Lévinas, l'attitude familière du jeu se dévoile comme légèreté, irréalité, ilôt de rêve surgi au sein du néant, évanouissement. A l'instar du rêve, le jeu se révèle évanescence gratuite ${ }^{10}$. Il implique pure dépense ${ }^{11}$, liberté ${ }^{12}$, liberté sans responsabilité ${ }^{13}$, jouissance de la vie ${ }^{14}$, frivolité fallacieuse... ${ }^{15}$.

Il faut avoir présent à l'esprit l'ensemble de ces caractères quand on rencontre chez Lévinas la métaphore du jeu. Voyons de plus près à quelle expérience existentielle - à quelle absence peuvent nous introduire des expressions telles que: jeu de nos relations avec le monde, jeu de la pensée qui re-présente, jeu ambigu de la sensibilité.

\section{B. Jeu des relations avec le monde}

Comment Lévinas peut-il parler du « jeu perpétuel de nos relations avec le monde " ? ${ }^{16} \mathrm{La}$ vie serait-elle un jeu? Ne seraitelle que légèreté, irréalité, ilôt de rêve surgi au sein du néant, évanouissement, évanescence, gratuité, pure dépense, liberté sans responsabilité, jouissance de la vie, frivolité fallacieuse...?

La jouissance de la vie, dit Lévinas, est jeu:

Vivre, c'est jouer en dépit de la finalité et de la tension de l'instinct; vivre de quelque chose sans que ce quelque chose ait le sens d'un but ou d'un moyen ontologique, simple jeu ou jouissance de la vie. Insouciance à l'endroit de l'existence qui a un sens positif. Elle consiste

9. E. LEvinas. De l'existence à l'existant. Paris, Vrin, 1947, (réédition 1981), p. 34-35.

10. Ibid. P. 170; voir aussi E. LÉvinas. Autrement qu'être ou Au-delà de l'essence. La Haye, M. Nijhoff, 1974, p. 209; E. LÉvinas, Humanisme de l'autre Homme. Montpellier, Fata Morgana, 1971, p. 43.

11. E. LÉVINAS. En découvrant l'existence avec Husserl et Heidegger. Paris, Vrin, 1949, p. 191; voir aussi Humanisme de l'autre Homme. Op. cit., p. 42.

12. E. LEvinas. Totalité et Infini: essai sur l'extériorité - La Haye, M. Nifhoff, 1961, p. 102.

13. E. LEvinas. Autrement qu'être... Op. cit., p. 161 ; p. 154, n. 2.

14. E. LEvinas. Totalité et Infini. Op. cit., p. 107.

15. E. LEvinas. Autrement qu'être... Op. cit., p. 6.

16. E. LEvinas. De l'existence à l'existant. Op. cit., p. 26 
à mordre à pleines dents aux nourritures du monde, à agréer le monde comme richesses, à faire éclater son essence élémentale. (...) Dans la jouissance, je suis absolument pour moi. Egoïste sans référence à autrui - je suis seul sans solitude, innocemment égoïste et seul. Pas contre les autres, pas «quant à moi » - mais entièrement sourd à autrui, en dehors de toute communication et de tout refus de communiquer - sans oreilles comme ventre affamé ${ }^{17}$.

Dans toute jouissance inhérente aux nourritures du monde se joue le jeu de l'être: le Même s'y complaît, «athée, chez soi, séparé, heureux, créé...» ${ }^{18}$. L'identité de l'être s'y maintient dans le jeu normal de l'action et de la passion ${ }^{19}$.

Le jeu s'inscrit également dans les relations avec autrui. Lévinas conteste que la relation avec l'autre soit fusion ${ }^{20}$. La volupté, il est vrai, «n'est pas un plaisir comme un autre, parce qu'elle n'est pas un plaisir solitaire comme le manger et le boire» 21 .

La caresse est un mode d'être du sujet, où le sujet dans le contact d'un autre va au-delà de ce contact. Le contact en tant que sensation fait partie du monde de la lumière. Mais ce qui est caressé n'est pas touché à proprement parler. Ce n'est pas le velouté ou la tiédeur de cette main donnée dans le contact que cherche la caresse. Cette recherche de la caresse en constitue l'essence par le fait que la caresse ne sait pas ce qu'elle cherche. Ce « ne pas savoir», ce désordonné fondamental en est l'essentiel. Elle est comme un jeu avec quelque chose qui se dérobe, et un jeu absolument sans projet ni plan, non pas avec ce qui peut devenir nôtre et nous, mais avec quelque chose d'autre, toujours autre, toujours inaccessible, toujours à venir ${ }^{22}$.

Dans l'érotique, « les relations avec autrui se jouent - on joue avec autrui comme avec un jeune animal » ${ }^{23}$. Dans la relation érotique

... se joue le jeu des possibilités du moi, et dans ce jeu, sous les espèces du moi, se produit l'origine dans l'être. L'être ne s'y produit pas comme le définitif d'une totalité, mais comme un recommencement incessant et, par là, comme infini. Mais, dans le sujet, la production de

17. E. LÉvinas. Totalité et Infini. Op. cit., p. 107.

18. Ibid. P. 121.

19. Voir E. LEvinas. Autrement qu'être... Op. cit., p. 146.

20. Voir E. LEvinas. Le Temps et l'Autre. Paris, P.U.F., 1983, p. 83.

21. Ibid. P. 82.

22. Ibid. Voir aussi Totalité et Infini. Op. cit., p. 235 et suiv.

23. E. LÉvinas. Totalité et Infini. Op. cit., p. 241. 
l'origine est production de vieillesse et de mort qui se joue du pouvoir. Le moi revient à soi, se retrouve le Même, malgré tous ses recommencements, retombe solitaire sur ses pieds, ne dessine qu'un destin irréversible. La possession de soi devient l'encombrement par soi ${ }^{24}$.

Aussi l'amour érotique oscille-t-il « entre l'au-delà du désir et l'en deça du besoin"; « sa jouissance prend place parmi tous les autres plaisirs et joies de la vie» ${ }^{25}$.

Lorsque tout «s'absorbe, s'enlise et s'emmure dans le Même » ${ }^{26}$, l'enchantement est fragile. L'ennui menace, et ne fautil pas redouter, selon les mots de Lévinas, "une hébétude, une pétrification ou un engraissement ou une paresse " ${ }^{27}$. Car, dans l'enchaînement à lui-même, "le Moi étouffe en Soi, de par la façon tautologique de l'identité, et recherche sans cesse la distraction du jeu et du sommeil» ${ }^{28}$.

En plus du jeu des relations avec le monde s'offre la possibilité «des jeux internes de l'âme» ${ }^{29}$, c'est-à-dire des jeux de l'art, du savoir scientifique et philosophique, en bref, de la pensée qui re-présente.

\section{Jeu de la pensée qui re-présente}

Une grande partie de l'œuvre de Lévinas comporte la critique de la philosophie occidentale qui, écrit le philosophe, "coïncide avec le dévoilement de l'Autre où l'Autre, en se manifestant comme être, perd son altérité » ${ }^{30}$. Essentiellement une philosophie de l'être, la philosophie occidentale fait de la compréhension de l'être «son dernier mot et la structure fondamentale de l'homme» ${ }^{31}$. Lévinas dénonce cette philosophie de l'immanence et de l'autonomie ou athéisme. «Le Dieu des philosophes, d'Aristote à Leibniz, à travers le Dieu des scolastiques - est un dieu adéquat à la raison, un dieu compris qui ne saurait

\footnotetext{
24. Ibid. P. 248.

25. Ibid. P. 249.

26. E. Levinas. De Dieu qui vient à l'idée. Op. cit., p. 31.

27. Ibid. P. 60.

28. E. LÉvinas. Autrement qu'être... Op. cit., p. 160.

29. E. LEvinas. En découvrant l'existence... Op. cit., p. 174.

30. Ibid. P. 188.

31. Ibid.
} 
troubler l'autonomie de la conscience, se retrouvant elle-même à travers toutes ses aventures, retournant chez soi comme Ulysse qui, à travers toutes ses pérégrinations ne va que vers son île natale» ${ }^{32}$.

Convaincu que «le domaine du sensé n'est pas limité au sérieux ${ }^{33}$ des sciences et des travaux qui s'attachent à l'être thématisé, ni au jeu des plaisirs et des arts, qui s'évade de l'être, mais en conserve le souvenir, se complaît dans ses images et comporte des enjeux " ${ }^{34}$, Lévinas dénonce l'illusion que comporte ce qu'il appelle les «jeux de l'intériorité» ${ }^{35}$. Toute philosophie qui «tient à résorber tout Autre dans le Même et à neutraliser l'altérité», toute "Action d'avance récupérée dans le savoir qui la guide», tout art, dans le jeu infini duquel «l'être s'allège de son altérité» ${ }^{36}$, toute expérience qui, "si passive qu'elle soit, si accueillante qu'elle soit, se convertit aussitôt en "constitution de l'être" qu'elle reçoit, comme si le donné était tiré de soi, comme si le sens qu'il apporte était prêté par (le) moi» ${ }^{37}$, se tiennent au bord de l'illusion. "L'ombre est prise pour une proie, la proie est lâchée pour l'ombre» ${ }^{38}$.

Car la connaissance est en somme une relation de soi à soi ${ }^{39}$. L'intentionnalité théorétique ramène l'objet à la conscience et l'instaure en elle; la conscience ordonne ses concepts et les déroule dans la vérité. Les présupposés de la vérité, écrit Lévinas, «telles des conventions, apparentent la combinaison des concepts à un jeu» ${ }^{40}$. "La connaissance repose sur l'apparition, sur le phénomène, lequel déroule l'être de l'étant, mettant, par la lumière, toutes choses ensemble, ordonnant l'ordre. Prises dans la lumière, inévitablement contemporaines, les choses sont présentes

32. Ibid.

33. E. LÉviNAS reconnaît sans aucun doute le "sérieux» des sciences et de l'ontologie traditionnelle. Mais l'ensemble de son cuvre indique que, contrairement à l'opinion courante, le véritable sérieux, la véritable gravité ne se trouvent ni dans les sciences, ni dans la philosophie occidentale. Voir le deuxième point de la présente étude.

34. E. Lévinas. De Dieu qui vient à l'idée. Op. cit., p. 194.

35. E. LEvinas. En découvrant l'existence... Op. cit., p. 188.

36. E. LÉvinAs. Humanisme de l'Autre Homme. Montpellier, Fata Morgana, 1971, p. 40.

37. E. LEvinas. En découvrant l'existence... Op. cit., p. 188.

38. Ibid.

39. Voir E. LÉvinas. Autrement qu'être... Op. cit., p. 135.

40. Ibid. P. 74. 
jusque dans leurs cachettes les plus secrètes, comme si l'être était une partie de colin-maillard où le bandeau sur les yeux n'empêche pas la présence de vous héler de partout ${ }^{41}$. Jeu de la connaissance qui se joue au présent : « jeu de clarté et d'abscondité» ${ }^{42}$ au cours duquel se dévoile l'être, approche qui se polarise en corrélation sujet-objet ${ }^{43}$, «ambitieuse entreprise philosophique en faveur de la pensée et contre le pur calcul, mais subordonnant l'humain aux jeux anonymes de l'Être» ${ }^{44}$.

«Ce jeu dans l'être est la conscience même: présence à soi par la différence qui est, à la fois, la perte de soi et les retrouvailles dans la vérité. Le pour soi de la conscience est ainsi le pouvoir même que l'être exerce sur lui-même, sa volonté, sa principauté. Il s'y égale à soi et se possède. La domination est dans la conscience comme telle...» ${ }^{45}$. Car le donné se présente d'abord à distance de la conscience, se re-présente, laissant l'intervalle de l'espace et du temps nécessaire à l'accueil; puis la conscience identifie et possède; elle s'illumine «en s'interrompant et en se retrouvant dans le jeu temporel des rétentions et des protentions ${ }^{46}$, par lesquelles tout présent est re-présentation» ${ }^{47}$. Le soi semble issu de sa propre initiative; dans les jeux et les figures de la conscience, il paraît coïncider avec lui-même « libre en tant que totalité ne laissant rien au dehors, et, ainsi, pleinement raisonnable, il se pose comme terme toujours convertible en relation: conscience de soi» ${ }^{48}$.

Cette retraite dans "le jeu que l'être joue dans la conscience ${ }^{49}$ protège le processus de l'objectivité ou de «l'essence de l'être révélée dans la vérité» ${ }^{50}$. Cependant la subjectivité du sujet pensant est menacée par l'objectivité ou le «train de l'essence». "Cette possibilité d'absorber le sujet, écrit Lévinas, auquel l'essence se confie, est le propre de l'essence. Tout s'enferme en elle.

41. E. Levinas. En découvrant l'existence... Op. cit., p. 214.

42. Ibid. P. 216.

43. Voir lbid. P. 204.

44. E. Levinas. Difficile Liberté: essais sur le judaïsme. Paris, Albin Michel, 1963, p. 360 .

45. E. LEvinas. Autrement qu'être... Op. cit., p. 129.

46. Ibid. P. 132.

47. Ibid. P. 133.

48. Ibid. P. 133-134.

49. Ibid. P. 135.

50. Ibid. P. 167. Le mot « processus» indique bien ici le sens verbal de l'être. 
La subjectivité du sujet consisterait toujours à s'effacer devant l'être, à le laisser être en rassemblant les structures en signification, en proposition globale dans un Dit, en un grand présent de la synopsie où l'être brille de tout son éclat ${ }^{51}$. Le philosophe précise : "L'être - malgré ou à cause de sa finitude - a une essence englobante, absorbante, emmurante. La véracité du sujet n'aurait d'autre signification que cet effacement devant la présence, que cette représentation ${ }^{52}$. «Aveu de l'ultimité de l'Essence, de l'immanence sans issue de son jeu clôturant» ${ }^{53}$. Jeu. Rêve. Illusion. Liberté sans responsabilité.

Le Jeu de l'Essence se prolonge dans celui du Dit et des significations culturelles. Le processus, fût-il verbe, fût-il mouvement, "se montre, mais s'immobilise et se fixe dans le Dit » ${ }^{54}$. Il donne naissance à l'ontologie 55 . Mais le Dit épuise-t-il les possibilités de la signification? Y a-t-il un au-delà du Dit, un au-delà de l'Essence, un Dire dont la différence avec le Dit et l'Essence ne se réduirait pas à « un jeu frivole de la syntaxe» ? ${ }^{56} \mathrm{Y}$ a-t-il un terme possible au jeu du dévoilement de l'être «où toujours l'immanence gagne sur la transcendance - car une fois l'être dévoilé - fût-ce partiellement, fût-ce dans le mystère - il devient immanent» ${ }^{57}$. Y a-t-il possibilité de rompre avec «les jeux internes de l'âme » ${ }^{58}$, possibilité d'une direction « qui échappe au jeu bi-polaire de l'immanence et de la transcendance, propre à l'être, où l'immanence gagne, à tout coup, contre la transcendance»? 59

Emmanuel Lévinas a, en effet, repéré une autre voie, une direction vers le face à face, qui mène à son tour à une irrectitude radicale, mais avant d'accéder à l'une et à l'autre, nous devons porter notre réflexion sur le jeu ambigu de la sensibilité et sur la distraction du sommeil.

51. Ibid. P. 171.

52. Ibid.

53. Ibid. P. 222.

54. Ibid. P. 29.

55. Ibid. P. 55.

. 56. Ibid.

57. E. LÉvinas. «Un Dieu Homme?», dans Exercices de la patience. Op. cit., p. 71.

58. E. LÉvinAs. En découvrant l'existence... Op. cit., p. 174.

59. E. Lévinas. Humanisme de l'Autre Homme. Op. cit., p. 59 ; voir aussi En découvrant l'existence... Op. cit., p. 199. 


\section{Jeu ambigu de la sensibilité}

Le jeu perpétuel de nos relations avec le monde qui se joue tout spécialement dans la jouissance des biens de la vie et dans les rapports érotiques avec autrui, ainsi que le jeu de la raison qui re-présente (dans la science, dans l'art, dans la philosophie), conduisent tous à l'immanence du Moi emmuré en lui-même. La sensibilité pourrait-elle constituer un chemin vers le dehors, une ouverture par où pourrait s'engouffrer une bouffée d'air pour le Même, englobé dans la totalité de l'Être?

Lévinas décrit la sensibilité, «non pas comme un moment de la représentation, mais comme le fait de la jouissance» ${ }^{60}$. "Sentir, écrit-il, c'est précisément se contenter sincèrement de ce qui est senti, jouir, se refuser aux prolongements inconscients, être sans pensée, c'est-à-dire, sans arrière-pensées, sans équivoque, rompre avec toutes les implications - se sentir chez-soi» ${ }^{61}$. Cependant, dans le sensible comme vécu, l'identité se montre, l'essence "résonne»; la sensibilité joue, comme conscience, «son jeu logique et ontologique»; mais "jeu qui ne commence pas parcaprice», "qui ne déjoue pas les responsabilités qui le suscitent " ${ }^{62}$. La sensibilité ne se résorbe pas en « jeux de lumière et de reflets, ne s'écoule pas comme le temps remémorable de la conscience. Elle est vulnérabilité, susceptibilité, dénudation, cernée et concernée par autrui, irréductible à l'apparaître d'autrui» ${ }^{63}$. C'est parce qu'elle est jouissance qu'elle peut ainsi être vulnérabilité ou exposition à autrui ${ }^{64}$. C'est parce qu'elle est contact et vulnérabilité qu'elle peut ne jouer aucun jeu, interrompre «la plaisance et la complaisance du jeu» ${ }^{65}$. Car "seul le sens d'autrui est irrécusable et interdit la réclusion et la rentrée dans la coquille du soi. Une voix vient de l'autre rive» ${ }^{66}$ et interrompt la distraction du jeu.

Mais avant de prêter l'oreille à cette voix, il nous faut encore examiner la possibilité du sommeil, autre distraction à laquelle se

60. E. Lévinas. Totalité et Infini. Op. cit., p. 109.

61. Ibid. P. 112.

62. E. LÉvinas. Autrement qu'être... Op. cit., p. 46.

63. Ibid. P. 101-102.

64. Ibid. P. 93.

65. Ibid. P. 72 .

66. Ibid. P. 230 . 
livre le Même et que dénonce Emmanuel Lévinas. Cherchons le sens de cette deuxième métaphore.

\section{La métaphore du SOMMEIL}

\section{A) Pbénoménologie du sommeil}

Le sommeil, écrit Lévinas, "est précisément l'événement sans événement, l'événement intérieur ${ }^{67}$. «Dormir, c'est suspendre l'activité psychique et physique» ${ }^{68}$. Pour pouvoir suspendre l'activité, il faut un lieu; il faut «borner l'existence» à ce lieu, à cette position, établir une « relation avec le lieu comme base. En nous couchant, en nous blottissant dans un coin pour dormir, nous nous abandonnons à un lieu - il devient notre refuge en tant que base» ${ }^{69}$.

Le sommeil est donc reposition, repos, abandon au lieu comme refuge, suspension de l'être sans destruction. "C'est à partir du repos, à partir de la position, à partir de cette relation unique avec le lieu, que vient la conscience» ${ }^{70}$.

Comment la conscience se comporte-t-elle dans cette suspension de l'activité physique et psychique qu'implique le sommeil? Lévinas décrit la conscience comme « un engagement dans l'être qui consiste à se tenir précisément dans le non-engagement du sommeil» ${ }^{71}$. Tout ce qu'elle «a», mais qui est une condition sine qua non de son existence, c'est un lieu, une position : la conscience est ICI. «Dans sa vie même de conscience, elle vient toujours de sa position, c'est-à-dire de la "relation» préalable avec la base, avec le lieu que dans le sommeil, elle épouse exclusivement ${ }^{72}$.

Sa position est celle du corps : celui-ci est, par le lieu qui est une base, "l'avènement même de la conscience» ${ }^{73}$. C'est donc par la position que la conscience participe au sommeil. Lévinas écrit :

67. E. Lévinas. De l'existence à l'existant. Op. cit., p. 118.

68. Ibid. P. 119.

69. Ibid.

70. Ibid. P. 120.

71. Ibid.

72. Ibid. P. 122.

73. Ibid. 
«[1]a possibilité de reposer, de s'envelopper en soi, c'est la possibilité de s'abandonner à la base, de se coucher. Elle est contenue dans la conscience en tant que la conscience est localisée. Le sommeil, repli dans le plein, s'accomplit dans la conscience comme position. Mais la position est l'événement même de l'instant comme présent ${ }^{74}$.

Modalité de l'être, "quand l'être se retire de lui-même et quand il se libère de sa propre emprise sur lui-même» ${ }^{75}$, le sommeil porte en lui-même son échec. «Le sommeil fragile, sommeil aux ailes légères; est un état second ${ }^{76}$.

Quand l'être, dans le sommeil, se libère de sa propre emprise sur lui-même, sa liberté n'est pas un « néantissement». Mais cette liberté, dit Lévinas, n'est que «pensée» ${ }^{77}$. Qu'est-ce à dire? Lévinas s'en explique :

C'est cela la pensée de la liberté qui n'est que pensée: le recours au sommeil, à l'inconscience, fugue et non pas évasion; divorce illusoire entre moi et soi qui finira par une reprise de l'existence en commun; liberté qui ne suppose pas un néant où elle se jette, qui n'est pas comme chez Heidegger un événement de néantissement, mais qui se fait dans le "plein» même de l'être par la situation ontologique du sujet. Mais, espoir seulement de la liberté et non point liberté à l'égard de l'engagement $/ . . . /{ }^{78}$.

L'analyse phénoménologique de l'événement naturel du sommeil à laquelle se livre Lévinas, dans De l'existence à l'existant, en met en lumière les principales caractéristiques que nous devons retrouver en tout ou en partie dans l'emploi métaphorique du mot appliqué au psychisme: événement sans événement, reposition, repos, abandon au lieu comme refuge, suspension de l'être sans anéantissement, repli dans le plein, non-engagement, état second qui porte en lui son échec, fugue de la conscience, instant comme présent qui ne se réfère qu'à soi, liberté illusoire qui s'emprisonne dans une identification. En bref, distraction comme le jeu.

74. Ibid. P. 124. Suit ce texte une analyse du temps; ce n'est pas ici le lieu d'en rendre compte. Voir p. 124 et suiv., en particulier, p. 135.

75. Ibid. P. 142.

76. Ibid.

77. Ibid.

78. Ibid. P. 152. 


\section{B) Signification ontologique du sommeil}

La métaphore du sommeil devrait nous mettre sur la piste d'une "signification ontologique» ${ }^{79}$ particulière. Analysons les trois aspects suivants: le pouvoir de sommeil de la conscience, la position de repos du Même et la substantification de l'être qui lui est corrélative.

La conscience a «le pouvoir de "suspendre" l'être par le sommeil et l'inconscience» ${ }^{80}$, "pouvoir d'évanescence» ${ }^{81}$, "de détente, d'assoupissement, d'absence» ${ }^{82}$, pouvoir de trouver « un refuge en soi pour s'y retirer de l'être» et d'«avoir, comme Pénélope, une nuit à soi pour défaire l'ouvrage veillé et surveillé dans la journée ${ }^{83}$. Pouvoir de sombrer dans l'inconscience du sommeil qui «n'est pas une nouvelle vie qui se joue sous la vie: c'est une participation à la vie par la non-participation, par le fait élémentaire de reposer ${ }^{84}$. Évasion en soi. Oubli. Arrière-fond d' «inconscient», de «sommeil» et de mystère ${ }^{85}$. Possibilité de s'arracher à la vigilance, vigilance adossée à une possibilité de sommeil. "En fait, écrit Lévinas, la conscience participe déjà à la vigilance. Mais ce qui la caractérise spécialement, c'est de conserver toujours la possibilité de se retirer "derrière", pour dormir. La conscience a le pouvoir de dormir. Cette fuite dans le plein est comme le paradoxe même de la conscience» ${ }^{86}$.

Le Même en plein repos coïncide avec lui-même. Raison, il repose dans la lucidité de l'évidence ${ }^{87}$. Perfection du connaître. Ses figures sont intelligence et signifiance, mais intelligibilité qui s'assoupit, s'embourgeoise dans la présence satisfaite de son lieu ${ }^{88}$. Quiétude. Rêve dans son présent. Le Même se noyaute en substance, en identité, en repos, en présence, en sommeil ${ }^{89}$.

\footnotetext{
79. Ibid. P. 37.

80. Ibid. P. 43, 105.

81. Ibid. P. 110.

82. Ibid. P. 110-111.

83. Ibid. P. 110.

84. Ibid. P. 118-119.

85. Voir Ibid. P. 134, 171.

86. E. LEvinas. Le Temps et l'Autre. Op. cit., p. 30.

87. Voir E. LÉvinas. De Dieu qui vient à l'Idée. Op. cit., p. 61 ; Autrement qu'être... Op. cit., p. 186.

88. Voir E. LÉvinas. De Dieu qui vient à l'Idée. Op. cit., p. 57, 58.

89. Voir Ibid. P. 99.
} 
Nous voici conduits au cœur d'une ontologie où l'être est substantif. La substance suppose l'identification même de l'identique ou encore la non-inquiétude de l'identité, comme l'acte de son repos ${ }^{90}$. Paradoxale contradiction, puisque l'être, verbe des verbes, a été pensé par les grecs comme acte pur. Comment tirer le Même de son repos, de sa quiétude? Comment sortir d'une ontologie qui re-pose sur l'évidence du savoir?

Lévinas indique une route qui conduit au dégrisement ou au «réveil» secouant le «sommeil dogmatique» qui se dort au fond de toute conscience reposant sur l'objet» ${ }^{91}$; sur cette route, il y a un événement : le surgissement d'Autrui dans l'horizon du Même. Nous emprunterons ce chemin en analysant, dans un deuxième point, la métaphore de la GRAVITÉ et celle de l'INSOMNIE.

Auparavant, rappelons que les métaphores du JEU et du SOMMEIL s'appliquent à dénoncer les distractions d'un Moi qui ajourne indéfiniment sa prise de responsabilité. Réfugié dans la quiétude du sommeil, aux frontières de l'inconscience, ou encore maître de son jeu de concepts, le Même se satisfait dans la jouissance de la vie, dans une liberté sans responsabilité, dans l'irréalité et l'illusion, dans la "semblance» du savoir, dans le repli en soi, dans son pouvoir d'oubli, d'évanescence. «Le rêve et l'illusion, écrit Lévinas, - c'est le jeu d'une conscience sortie de l'obsession, touchant l'autre sans être assigné par lui. Jeu de la conscience - semblance» ${ }^{92}$.

\section{VERS LA GRAVITE DU «POUR-AUTRUI» ET L'INSOMNIE DE LA RESPONSABILITÉ ÉTHIQUE}

Si la vie n'est que jeu, c'est-à-dire légèreté, irréalité, ilôt de rêve surgi au sein du néant, éva nescence gratuite; si la conscience a le pouvoir de sommeiller dans l'irresponsabilité du nonengagement, l'existence n'est-elle pas en définitive un fiasco, la «vanité des vanités»? «Mais, écrit Lévinas, le fiasco de l'humain qui nous paraît surgir dans le prolongement d'une certaine exaltation du Même, de l'Identique, de l'Activité et de l'Être - et ne fût-ce que comme leur remise en question -, ne suggère-t-il

90. Voir Ibid. P. 78, 175.

91. Ibid. P. 105.

92. E. LEvinas. Autrement qu'être... Op. cit., p. 110, n. 21. 
pas, par cette remise en question même, une autre signifiance : un autre sens et une autre façon de signifier ? ${ }^{93}$.

La lassitude elle-même qui frappe l'existence, n'est-elle pas en somme comme « un rappel d'un engagement à exister, de tout le sérieux, de toute la dureté d'un contrat irrésiliable»? ${ }^{94}$. Sens désaccordé à l'Être où échoue la rationalité du Même ${ }^{95}$. Sens nouveau qui vient du surgissement d'Autrui. "Seul le sens d'autrui est irrécusable et intérdit la réclusion et la rentrée dans la coquille du soi. Une voix vient de l'autre rive ${ }^{96}$, disions-nous plus haut. Elle interrompt la gravitation égoïste du Même. Elle porte en elle toute la gravité de la PROXIMITÉ, l'obsession, l'assignation à l'insomnie, c'est-à-dire à la responsabilité.

\section{Gravité de la PROXIMITÉ}

La jouissance noyaute le Même. L'immédiateté du sensible « est d'abord l'aisance du jouir, (...) dans sa fraîcheur incomparable de plénitude et de comblement - plaisir (...). Complaisance de la subjectivité, complaisance éprouvée pour elle-même - ce qui est son «égoïté» même, sa substantialité. Mais aussitôt «dénucléation» du bonheur imparfait qui est le battement de la sensibilité » ${ }^{97}$.

Car «l'immédiateté à fleur de peau de la sensibilité» 98 est aussi sa vulnérabilité. Exposition à la jouissance, elle est aussi exposition à la blessure, passivité, patience. C'est dans la vulnérabilité que la sensibilité prend SENS; elle SIGNIFIE: «par l'autre et pour l'autre: pour autrui. Non pas dans les sentiments élevés dans "les belles lettres", mais comme lors d'un arrachement du pain à la bouche qui le savoure, pour le donner à l'autre, la dénucléation de la jouissance où se noyaute le noyau du Moi ${ }^{99}$. En d'autres termes, dans la passivité, dans la patience de la vulnérabilité, exposé à l'Autre, le Même « perce la croûte de son

93. E. LÉvinas. De Dieu qui vient à l'Idée. Op. cit., p. 86.

94. E. LÉvinas. De l'existence à l'existant. Op. cit., p. 31.

95. Voir E. LEvinas. De Dieu qui vient à l'Idée. Op. cit., p. 86.

96. E. LEvinas. Autrement qu'être... Op. cit., p. 230.

97. Ibid. P. 81.

98. Ibid. P. 80 .

99. Ibid. P. 81 
égoïsme et comme déplace le centre de sa gravité hors [de lui] pour vouloir comme Désir et Bonté que rien ne limite» ${ }^{100}$. Bonté libérée de la gravitation égoïste, existence pour Autrui, Désir de l'Autre.

S'il y a un ordre qui tranche sur les choses sérieuses et même où le sérieux manque totalement — l'ordre du jeu et du sommeil l'ordre où « l'être, la totalité, l'État, la politique, les techniques, le travail, sont à tout moment sur le point d'avoir leur centre de gravitation en eux-mêmes, de peser pour leur compte» ${ }^{101}$, il en existe un autre d'une gravité extrême, où le Même aurait pu rester spectateur, mais dans lequel il devient responsable. Dans cet ordre, « rien n'est plus théâtre, le drame n'est plus jeu. Tout est grave» ${ }^{102}$.

C'est ici que la pensée d'Emmanuel Lévinas déconcerte le plus.

Pourquoi Autrui me concerne? Que m'est Hécube? Suis-je le gardien de mon frère? - ces questions n'ont de sens que si on a déjà supposé que le Moi n'a souci que de soi, n'est que souci de soi. Dans cette hypothèse, en effet, il reste incompréhensible que le hors-de-Moi absolu - Autrui - me concerne. Or, dans la «préhistoire» du Moi posé pour soi, parle une responsabilité. Le soi est de fond en comble otage, plus anciennement que Égo, avant les principes. Il ne s'agit pas pour le Soi, dans son être, d'être. Au-delà de l'égoïsme et de l'altruisme, c'est la religiosité de soi ${ }^{103}$.

Que veut dire le philosophe? Autrui, c'est en bref, la présence d'un être qui n'entre pas dans la sphère du Même. Il arrive du dehors, séparé - ou saint - visage. Il se pose en face du Même, comme infini, absolument autre. Son extériorité est un appel qui ne limite pas la liberté du Même. Au contraire, en l'appelant à la responsabilité, il l'instaure et la justifie. "La "résistance" de l'Autre, écrit Lévinas, ne me fait pas violence, n'agit pas négativement : elle a une structure positive : éthique. La première révélation de l'autre, supposée dans toutes les autres relations avec lui, ne consiste pas à le saisir dans sa résistance

100. E. LEvinas. Totalité et Infini. Op. cit., p. 217.

101. E. LEVINAS. Autrement qu'être... Op. cit., p. 203.

102. E. LEVINAS. Humanisme de l'Autre Homme. Op. cit., p. 79.

103. E. ĹEvinas. Autrement qu'être... Op. cit., p. 150. 
négative, et à le circonvenir par la ruse. Je ne lutte pas avec un dieu sans visage, mais réponds à son expression, à sa révélation» 104 .

Le visage ne s'offre pas aux pouvoirs du Même, à la possession. Il s'y refuse. Résistance totale à la prise. Le Visage parle. Il invite le Même «à une relation sans commune mesure avec un pouvoir qui s'exerce, fût-il jouissance ou connaissance» ${ }^{105}$. C'est pourquoi la relation avec le Visage quitte l'ordre du jeu et entre dans celui du sérieux et de la gravité.

Dans le face à face, le Visage ne se résorbe pas dans la thématisation, c'est-à-dire dans la représentation et le savoir :

Entendre sa misère qui crie justice ne consiste pas à se représenter une image, mais à se poser comme responsable, à la fois comme plus et comme moins que l'être qui se présente dans le visage. Moins, car le visage me rappelle à mes obligations et me juge. L'être qui se présente en lui vient d'une dimension de hauteur, dimension de la transcendance où il peut se présenter comme étranger, sans s'opposer à moi, comme obstacle ou ennemi. Plus, car ma position de moi consiste à pouvoir répondre à cette misère essentielle d'autrui, à me trouver des ressources. Autrui qui me domine dans sa transcendance est aussi l'étranger, la veuve et l'orphelin envers qui je suis obligé ${ }^{106}$.

L'épiphanie du Visage est donc pour Lévinas la source d'où surgit le SENS, la SIGNIFICATION véritable, c'est-à-dire le pour-autrui, l'un-pour-l'autre, l'autre-dans-la-peau. L'encerclement de la totalité rompu par l'excédent non résorbable de l'Infini du Visage, le Même dans la relation du face à face, se découvre être non pas un Moi, un Égo, mais un Soi, d'emblée à l'accusatif, sujet qui n'a pas le statut d'un principe : « [1]e Soi est Sub-jectum: il est sous le poids de l'univers - responsable de tout» ${ }^{107}$. Sujet qui ne jouit pas d'une "liberté sans responsabilité, d'une liberté de jeu», mais qui, dans l'obsession, assume « une responsabilité qui ne repose sur aucun engagement libre» ${ }^{108}$, antérieure à tout engagement préalable, pré-originelle. Cette responsabilité pour autrui

104. E. LEvinas. Totalité et Infini. Op. cit., p. 171.

105. Ibid. P. 172.

106. Ibid. P. 190.

107. E. LÉvinas, Autrement qu'être... Op. cit., p. 147.

108. Ibid. P. 148. 
à laquelle le Soi (ou le Sub-jectum) est assigné dans son incondition d'otage, c'est "la fraternité humaine elle-même, antérieure à la liberté» ${ }^{109}$, accusation absolue, constituant la liberté elle-même qui, alliée au Bien, « situe au-delà et en dehors de toute essence» ${ }^{110}$.

Autrement qu'être ou au-delà de l'essence: voilà où « la voix qui vient de l'autre rive» ${ }^{111}$, la voix de l'Autre, la voix de l'Infini a mené Emmanuel Lévinas. «Être-soi, autrement qu'être, se désintéresser, c'est porter la misère et la faillite de l'autre et même la responsabilité que l'autre peut avoir de moi ; être soi — condition d'otage - c'est toujours avoir un degré de responsabilité de plus, la responsabilité pour la responsabilité de l'autre» ${ }^{112}$.

Pourquoi cette condition ou in-condition d'otage? La relation éthique à laquelle oblige le Visage est au-delà de l'Être. «L'au-delà dont vient le visage est à la troisième personne» ${ }^{113}$. Lévinas le désigne par le pronom « $\mathrm{Il}$ » et parle de l'illéité de la troisième personne. Le visage se tient dans la trace de l'Illéité, car dans le visage, cette troisième personne s'est déjà retirée de toute révélation et de toute dissimulation. Elle a passé ${ }^{114}$. L'Illéité n'est pas un «moins que l'être».

Au contraire, le IL, «c'est toute l'énormité, toute la démesure, tout l'Infini de l'absolument autre, échappant à l'ontologie. La suprême présence du visage est inséparable de cette suprême et irréversible absence qui fonde l'éminence même de la visitation» ${ }^{115}$ ou de l'apparition du visage d'autrui.

Du jeu de l'essence qui se joue dans l'ontologie, Emmanuel Lévinas se détourne et lui substitue la gravité et le sérieux de l'Autrement qu'être. Laissons-lui une fois de plus la parole:

Le visage de l'autre dans la proximité - plus que représentation est trace irréprésentable, façon de l'Infini. Ce n'est pas parce que,

109. Ibid. P. 148-149.

110. Ibid. P. 150.

111. Ibid. P. 230.

112. Ibid. P. 149-150.

113. E. LÉvinas. Humanisme de l'Autre Homme. Op. cit., p. 59 ; voir aussi En découvrant l'existence... Op. cit., p. 199.

114. Ibid.

115. E. LEvinas. En découvrant l'existence... Op. cit., p. 199. 
parmi les êtres, existe un Moi, être poursuivant des fins que l'Être prend une signification et devient univers. C'est parce que dans l'approche s'inscrit ou s'écrit la trace de l'Infini - trace d'un départ, mais trace de ce qui, dé-mesuré n'entre pas dans le présent et invertit l'arché en anarchie - qu'il y a délaissement d'autrui, obsession par lui, responsabilité et Soi. Le non interchangeable par excellence, le Je, l'unique se substitue aux autres. Rien n'est jeu. Ainsi se transcende l'être» ${ }^{116}$.

La gravité extrême de la Proximité assigne le Soi à la vigilance et à l'insomnie.

\section{Insomnie et vigilance}

La récurrence de la métaphore de l'insomnie dans l'œuvre lévinassienne indique l'importance qu'y attache le philosophe. L'indicible se fraie un chemin dans cette négation du sommeil et l'éthique fracture et excède le discours philosophique par la charge positive que véhicule la négation qui «résonne» dans le vocable. Suivons le fil de la métaphore. De l'insomnie comme phénomène naturel, procédons au «catégorial de l'insomnie » ${ }^{117}$.

\section{A. L'insomnie, phénomène naturel}

Ces moments où le sommeil résiste à notre appel nous sont à tous, une nuit ou l'autre, familiers :

On veille quand il n'y a plus rien à veiller et malgré l'absence de toute raison de veiller. Le fait nu de la présence opprime: on est tenu à l'être, tenu à être. On se détache de tout objet, de tout contenu, mais il y a présence. Cette présence qui surgit derrière le néant n'est ni un être, ni le fonctionnement de la conscience s'exerçant à vide, mais le fait universel de l'il $y$ a, qui embrasse et les choses et la conscience ${ }^{118}$.

Lévinas ne dote pas le il y a du même contenu que le « il y a » heideggerien. Certains souvenirs d'enfance (il se rappelle avoir perçu le silence de sa chambre à coucher comme bruissant) et l'expérience de l'insomnie lui ont fait découvrir que le néant n'est pas l'unique négation de l'être. Dans l'obscurité de la nuit, les

116. E. LEvinas. Autrement qu'être... Op. cit., p. 149.

117. E. LEvinas. De Dieu qui vient à l'Idée. Op. cit., p. 98, 99.

118. E. LÉvinas. De l'existence à l'existant. Op. cit., p. 109. 
étants sont soustraits à la vision; mais le vide est plein de l'il y a. Présence invisible, "bruissement anonyme» ${ }^{119}$, présence dans l'absence, grouillement parfois horrifiant, qui coupe le souffle:

La vigilance est absolument vide d'objets. Ce qui ne revient pas à dire qu'elle est expérience du néant; mais qu'elle est aussi anonyme que la nuit elle-même. L'attention suppose la liberté du moi qui la dirige; la vigilance de l'insomnie qui tient ouverts nos yeux n'a pas de sujet. C'est le retour même de la présence dans le vide laissé par l'absence - non pas retour de quelque chose, mais d'une présence; c'est le réveil de l'il y a au sein de la négation (...). Ce retour de la présence dans l'absence ne se fait pas dans des instants distincts, comme un flux et un reflux. Le rythme manque à l'il y $a$, comme la perspective aux points grouillants de l'obscurité ${ }^{120}$.

Dans le phénomène de l'insomnie, Lévinas distingue l'une de l'autre la conscience et la veille. La conscience ne fait que participer à la veille, elle n'en est qu'une partie, c'est-à-dire : elle l'a déjà déchirée. La veille est anonyme. «Il n'y a pas ma vigilance à la nuit, dans l'insomnie, c'est la nuit elle-même qui veille. Ça veille. Dans cette veille anonyme où je suis entièrement exposé à l'être, toutes les pensées qui remplissent mon insomnie sont suspendues à rien. Elles sont sans support. Je suis, si l'on veut, l'objet plutôt que le sujet d'une pensée anonyme» ${ }^{121}$.

Lévinas insiste sur l'impersonnalité de l'il y a. Impersonnalité comme dans les expressions : «il pleut», «il fait nuit». Ni être, ni néant. Dans l'insomnie, "l'impossibilité de sortir de la veille est quelque chose "d'objectif", d'indépendant de mon initiative /.../. Je ne veille pas: "ça" veille» ${ }^{122}$.

À cette absence de maîtrise, s'ajoute la conscience « que cela ne finira jamais, c'est-à-dire qu'il n'y a plus aucun moyen de se retirer de la vigilance à laquelle on est tenu. (...) C'est toujours le même présent ou le même passé qui dure. (...) Seuls les bruits extérieurs qui peuvent marquer l'insomnie, introduisent des commencements dans cette situation sans commencement ni fin,

119. E. LEVINAS. Totalité et Infini. Op. cit., p. 236.

120. E. LEvinas. De l'existence à l'existant. Op. cit., p. 110-111.

121. Ibid. P. 111.

122. E. LÉvinAs. Éthique et Infini. Op. cit., p. 47; voir aussi Totalité et Infini. Op. cit., p. 27. 
dans cette immortalité à laquelle on ne peut échapper, toute semblable à l'il $y a$, à l'existence impersonnelle» ${ }^{123}$.

Le phénomène naturel de l'insomnie, tel que décrit dans l'analyse phénoménologique de Lévinas, offre les caractères suivants : un événement sans raison d'être, puisqu'il est vide d'objets ; un événement anonyme qui échappe à la maîtrise d'un sujet qui en est plutôt l'objet; exposition à une impossibilité de sommeil, de détente, d'assoupissement, d'absence; assignation à la veille, antérieure à la volonté, à la liberté. Passivité plus passive que toute passivité.

Il n'est donc pas étonnant que, chez Lévinas, l'insomnie puisse jouer le rôle de métaphore et, menant la pensée au cœur de la question de l'«autrement qu'être», l'ouvrir à la dimension du pré-originel : réalité plus réelle que toute réalité, source de toute éthique.

\section{B. Le catégorial de l'insomnie}

L'insomnie, tant au plan physique que métaphorique, est absence de repos, de quiétude; elle est l'in-quiétude par excellence. Elle ne s'inscrit cependant pas dans une liste de catégories où elle trouverait place à titre d'activité, c'est-à-dire comme un acte exercé par le Même sur l'Autre. Au contraire, c'est l'Autre qui tire le Même de son repos, de sa quiétude. Voyons comment Lévinas manie la métaphore de l'insomnie:

L'insomnie, - la veillée de l'éveil - est inquiétée du coeur de son égalité formelle ou catégoriale par l'Autre qui dénoyaute tout ce qui, en elle, se noyaute en substance du Même, en identité, en repos, en présence, en sommeil ; par l'Autre qui déchire ce repos, qui le déchire en l'en deça de l'état où l'égalité tend à s'installer. C'est précisément là le caractère catégorial irréductible de l'insomnie: l'Autre dans le Même qui n'aliène pas le Même, mais précisément l'éveille ; éveil comme exigence qu'aucune obédience n'égale, n'endort: un "plus» dans le «moins». (...) Passivité de l'Inspiration ou subjectivité du sujet dégrisé de son être. (...) Insomnie ou veille, mais veille sans intentionalité - dés-intéressée ${ }^{124}$.

123. E. LÉvinas. Le Temps et l'Autre. Op. cit., p. 27.

124. E. LEvinas. De Dieu qui vient à l'Idée. Op. cit., p. 98-99; voir aussi p. 130. 
Pour dire le psychique, Lévinas « remonte aux mots comme insomnie, ou veiller, ou vigilance» ${ }^{125}$, qui s'opposent à leur antithèse, le sommeil. Les deux métaphores, sommeil et insomnie, indiquent la double possibilité du psychisme: 1) ou bien se « reposer» dans la connaissance, dans le savoir, dans l'ontologie et jouer le jeu de l'art, des concepts et des relations ludiques avec le monde ; 2) ou bien, dans son repos, se laisser interpeler par la gravité de l'altérité, par l'inquiétude et l'insomnie qui «viennent de la misère des autres et où l'insomnie n'est que l'absolue impossibilité de se dérober et de se distraire ${ }^{126}$.

Lévinas multiplie les textes dans lesquels l'insomnie - ou le veiller - apparaît liée à « la scission de l'identité» 127 du Même; à "la dénucléation de l'atomicité même de l'un» ${ }^{128}$; au «désappointement de sa ponctualité même» ${ }^{129}$; au « déchirement qui est non pas la finitude d'un être incapable de se rejoindre et de «demeurer en repos» en guise d'état d'âme, mais transcendance déchirant ou inspirant l'immanence» ${ }^{130}$; à une "veille sans intentionalité mais seulement réveillée sans cesse de son état même de veille, se dégrisant de son identité pour le plus profond que soi» ${ }^{131}$.

Que SIGNIFIE cette vigilance, ce réveil dans l'éveil, sinon la rupture, la dé-fection de l'identité ou de l'égoïté du Même, scission qui n'est pas sa destruction, son néantissement, mais qui signifie sa substitution au prochain, à l'étranger, à l'apatride? Substitution qui instaure un ordre ou un désordre nouveaux « où la raison n'est plus ni connaissance ni action mais où désarçonnée par Autrui de son état - désarçonnée du Même et de l'être - elle est relation éthique avec autrui, proximité du prochain» ${ }^{132}$.

Proximité du prochain. Gravité. Insomnie. Approcher Autrui, c'est chercher celui que l'on a trouvé, c'est ne jamais être quitte envers lui; c'est non pas simple coexistence, mais inquiétude,

125. E. LÉvinAs. Transcendance et Intelligibilité. Op. cit., p. 39.

126. E. LEvinas. Autrement qu'être... Op. cit., p. 118.

127. E. Lévinas. De Dieu qui vient à l'Idée. Op. cit., p. 50.

128. Ibid. P. 51.

129. Ibid.

130. Ibid.

131. Ibid.

132. Ibid. P. 60. 
non-repos. C'est, bien plus, l'altérité dans l'identité, l'autre-dansla-peau, l'animation, le pneuma même du psychisme. C'est "l'identité d'un corps s'exposant à l'autre, se faisant "pour l'autre": la possibilité du donner» ${ }^{133}$. "La proximité, écrit Lévinas, comme le "de plus en plus proche" se fait sujet. Elle atteint son superlatif comme mon inquiétude incessible, se fait unique, dès lors un, oublie la réciprocité comme dans un amour qui n'attend pas de partage» ${ }^{134}$. C'est dans cet oubli de la réciprocité que Lévinas s'éloigne de Martin Buber et de Gabriel Marcel chez lesquels le Je et le $\mathrm{Tu}$ entretiennent des relations d'égalité et d'échanges réciproques. L'asymétrie de l'interpersonnel souligne l'originalité de la pensée lévinassienne.

La proximité est donc responsabilité pour le prochain, jusqu'à la substitution à lui ${ }^{135}$. La proximité n'est pas une confusion avec Autrui, mais elle est « signification pure», « signification incessante», in-quiétude pour l'autre, réponse tenue pour l'autre, responsabilité, incapacité de se taire ${ }^{136}$. C'est le SENS, la signification, «la signifiance même du Visage, du parler originaire qui me demande et me tient en question et m'éveille ou suscite ma réponse ou ma responsabilité» ${ }^{137}$. La signification, c'est la bonté, laquelle est loin d'être un penchant altruiste à satisfaire. Otage d'autrui, le moi «obéit à un commandement avant de l'avoir entendu, fidèle à un engagement qu'il n'a jamais pris, à un passé qui n’a jamais été présent. Veille — ou ouverture du soi» ${ }^{138}$, la bonté est insomnie.

Nous voici aux antipodes du jeu et du sommeil, au sein desquels le fait d'être libre n'entraîne pas de responsabilité. Nous voici face à notre condition de créature dans un monde sans jeu, dans la gravité d'une passivité sans fin qui ne s'assume par aucune activité. Le Moi, - ou plutôt le Soi, parce que d'emblée à l'accusatif, sans que précède le nominatif —, est obsédé par Autrui, assume des responsabilités qu'il n'a pas prises, contrairement au sujet qui contemple librement et se décide. Comme

133. E. LEvinas. Autrement qu'être... P. 87.

134. Ibid. P. 103.

135. Voir E. LÉvinas. De Dieu qui vient à l'Idée. Op. cit., p. 113.

136. Voir E. LÉvinas. Autrement qu'être... Op. cit., p. 182.

137. E. Levinas. De Dieu qui vient à l'Idée. Op. cit., p. 251.

138. Ibid. P. 113. 
accusé de ce qu'il n'a jamais fait, persécuté et rejeté en soi, il assume, il "prend sur soi», "dans l'incapacité absolue de se dérober à la proximité, au visage, au délaissement de ce visage, là où l'infini est aussi absence. Plus exactement: le surgissement de l'ipséité est le fait même de cette gravité dans l'être » ${ }^{139}$.

Non-indifférence de l'un-pour-l'autre qui place sur la route du Don et du Sacrifice. Les mots (ou le Dit) trahissent la réalité en la thématisant. Au-delà du Dit, bien au-delà des mots, c'est à entendre un Dire originel que nous convie Lévinas; c'est à une rupture, à un éveil :

... éveil du Moi par Autrui, de moi par l'Etranger, de moi par l'apatride, c'est-à-dire par le prochain qui n'est que prochain. Eveil qui n'est ni réflexion sur soi, ni universalisation; éveil qui signifie une responsabilité pour autrui, pour autrui à nourrir et à vêtir, ma substitution à autrui, mon expiation pour la souffrance et, sans doute, pour la faute d'autrui. Expiation à moi impartie sans dérobade possible et par laquelle s'exacerbe irremplaçable, au lieu de s'aliéner, mon unicité de moi.

Mais dans cette rupture et cet éveil et cette expiation et cette exacerbation, se déroule la divine comédie d'une transcendance pardelà l'ontologie ${ }^{140}$.

\section{Conclusion}

En parcourant l'histoire de la philosophie, Emmanuel Lévinas a observé l'insécurité qui affecte le domaine de la raison. Depuis les premiers pas de la philosophie, la raison, écrit-il, « en tant que modalité de la connaissance, aurait à se méfier de certains jeux qui l'ensorcellent. Elle serait tenue à la vigilance pour déjouer des illusions. Il ne faut pas dormir, il faut philosopher» ${ }^{141}$.

Et pourtant, la Raison a paradoxalement été considérée comme le hâvre le plus sûr abritant le sensé dont le sérieux s'inscrit dans les domaines des sciences et de la philosophie.

C'est avec une telle tradition que rompt Emmanuel Lévinas. À la thématisation de l'être, au mode théorétique de l'ontologie, à

139. E. LEvinas. En découvrant l'existence... Op. cit., p. 233.

140. E. LÉvinAs. Noms propres. Op. cit., p. 12.

141. E. Lévinas. De Dieu qui vient à l'Idée. Op. cit., p. 35. 
la totalité qui englobe l'immanence du Même, le philosophe oppose une idée de l'Infini qui dégrise le Même et l'éveille à la transcendance. Car, pour Lévinas, « [j] amais la philosophie partant de la présence de l'être, ne s'en réveillera ou ne dira le réveil en d'autres termes qu'en ceux du savoir » ${ }^{142}$, jamais elle ne pensera la veille "et le réveil dont vit la veille - comme Raison sans l'entendre dans la connaissance» ${ }^{143}$. La SIGNIFICATION que connaît la philosophie se situe au niveau du savoir - vrai ou erroné - et se réfère à l'être, corrélatif de ce savoir.

Emmanuel Lévinas, attentif à l'extrême vivacité de la vie, au dérangement du Même par l'Autre, propose un autre SENS, une autre SIGNIFICATION qu'il exprime dans une formule lourde de responsabilité : l'un-pour-l'autre, l'autre-dans-la-peau. « Responsabilité d'otage jusqu'à la substitution à l'autre homme - infinie sujétion» ${ }^{144}$. Responsabilité incessible, élection dans un Dire pré-originel, an-archique, qui poussent Lévinas à poser la question ultime: Ai-je le droit d'être? Est-ce que je ne prends pas la place de quelqu'un? "Question de sens qui ne se tourne vers aucune finalité naturelle, mais qui se perpétue dans nos étranges discours humains sur le sens de la vie, où la vie s'éveille à l'humanité. Question refoulée la plupart du temps et qui remonte à la pointe extrême de ce qu'on appelle parfois, à la légère, maladie» ${ }^{145}$.

Question du SENS de l'être: non pas au niveau de la compréhension ontologique de ce verbe, mais au niveau de l'éthique de la justice de l'être. On comprendra pourquoi, dans ce cas, Lévinas parle de l'éthique comme philosophie première: première, parce qu'avant tous nos discours sur l'être, il y a eu une élection, dans un passé immémorial, qui assigne le Même à la responsabilité pour Autrui, et l'établit en relation avec l'Illéité, puisque Autrui, qui est Visage et Parole, se tient dans la trace de l'Illéité.

On comprendra aussi pourquoi les métaphores que recèle le style de Lévinas ont une "résonance» éthique. Si nous sommes pressés de quitter le jeu des relations avec le monde et le jeu des

142. Ibid. P. 56

143. Ibid.

144. Ibid. P. 255.

145. Ibid. P. 257. 
concepts, de secouer le sommeil dogmatique des sciences, de la philosophie, de la «bonne conscience» satisfaite de son retour incessant à soi, c'est que le sérieux et la gravité du SENS de l'ÊTRE - entendu dans sa verbalité — obsèdent le SOI jusqu'à l'insomnie.

«Pensée difficile, presque inaudible: pensée in-actuelle. (...) Pensée difficile: pensée inouïe. (...) Pensée difficile: pensée d'exception", écrit Jacques Rolland en colligeant quelques notes de lecture sur l'œuvre de Lévinas ${ }^{146}$. Il n'est pas étonnant que nos premiers contacts avec celle-ci nous laissent déconcertés au sein de nos jeux et de notre sommeil.

Université du Québec à Rimouski

146. Jacques Rolland. «Penser au-delà», dans Exercices de la patience. Op. cit., p. 11-16. 